\title{
Performance of the infrared array camera (IRAC) for SIRTF during instrument integration and test
}

Joseph L. Hora, Giovanni G. Fazio, Lori E. Allen, Matthew L.N. Ashby, Pauline Barmby, et al.

Joseph L. Hora, Giovanni G. Fazio, Lori E. Allen, Matthew L.N. Ashby, Pauline Barmby, Lynne K. Deutsch, Jiasheng Huang, S. Thomas Megeath, Michael A. Pahre, Brian Michael Patten, Zhong Wang, Steven P. Willner, William F. Hoffmann, J. Eric Mentzell, Peter Eisenhardt, Daniel Stern, Varoujan Gorjian, William J. Glaccum, Mark Lacy, Sean Carey, Jason A. Surace, William T. Reach, Judith L. Pipher, William J. Forrest, Craig R. McCreight, "Performance of the infrared array camera (IRAC) for SIRTF during instrument integration and test," Proc. SPIE 4850, IR Space Telescopes and Instruments, (5 March 2003); doi: 10.1117/12.461791

Event: Astronomical Telescopes and Instrumentation, 2002, Waikoloa, Hawai'i, United States 


\title{
Performance of the Infrared Array Camera (IRAC) for SIRTF during instrument integration and test
}

\author{
Joseph L. Hora $^{* a}$, Giovanni G. Fazio ${ }^{a}$, Lori E. Allen ${ }^{a}$, Matthew L. N. Ashby ${ }^{a}$, Pauline Barmby ${ }^{a}$, \\ Lynne K. Deutsch, Jiasheng Huang ${ }^{\mathrm{a}}$, S. Tom Megeath ${ }^{\mathrm{a}}$, Michael A. Pahre ${ }^{\mathrm{a}}$, Brian M. Patten ${ }^{\mathrm{a}}$, \\ Zhong Wang ${ }^{\mathrm{a}}$, Steven P. Willner ${ }^{\mathrm{a}}$, William F. Hoffmann ${ }^{\mathrm{b}}$, J. Eric Mentzell ${ }^{\mathrm{c}}$, Peter Eisenhardt ${ }^{\mathrm{d}}$, \\ Daniel Stern ${ }^{\mathrm{d}}$, Varoujan Gorjian ${ }^{\mathrm{d}}$, William Glaccum ${ }^{\mathrm{e}}$, Mark Lacy ${ }^{\mathrm{e}}$, Sean Carey ${ }^{\mathrm{e}}$, Jason A. Surace ${ }^{\mathrm{e}}$, \\ William T. Reach ${ }^{\mathrm{e}}$, Judith L. Pipher ${ }^{\mathrm{f}}$, William J. Forrest ${ }^{\mathrm{f}}$, Craig R. McCreight ${ }^{\mathrm{g}}$ \\ ${ }^{\mathrm{a}}$ Harvard-Smithsonian Center for Astrophysics, ${ }^{\mathrm{b}}$ University of Arizona, ${ }^{\mathrm{c}} \mathrm{NASA} /$ Goddard Space Flight Center, ${ }^{\mathrm{d} J e t}$ \\ Propulsion Laboratory, ${ }^{\mathrm{e}} \mathrm{SIRTF}$ Science Center/Caltech, ${ }^{\mathrm{f}}$ University of Rochester, ${ }^{\mathrm{g}} \mathrm{NASA} / \mathrm{Ames}$ Research Center
}

\begin{abstract}
The Infrared Array Camera (IRAC) is one of three focal plane instruments in the Space Infrared Telescope Facility (SIRTF). IRAC is a four-channel camera that obtains simultaneous images at 3.6, 4.5, 5.8, and 8 microns. Two adjacent $5.12 \times 5.12$ arcmin fields of view in the SIRTF focal plane are viewed by the four channels in pairs (3.6 and 5.8 microns; 4.5 and 8 microns). All four detector arrays in the camera are $256 \times 256$ pixels in size, with the two shorter wavelength channels using InSb and the two longer wavelength channels using Si:As IBC detectors. We describe here the results of the instrument functional and calibration tests completed at Ball Aerospace during the integration with the cryogenic telescope assembly, and provide updated estimates of the in-flight sensitivity and performance of IRAC in SIRTF.
\end{abstract}

Keywords: infrared astronomy, infrared arrays, calibration, SIRTF, IRAC

\subsection{Description of IRAC}

\section{INTRODUCTION}

IRAC $^{1}$ is a four-channel camera that obtains simultaneous $5.12 \times 5.12$ arcmin images at $3.6,4.5,5.8$, and $8 \mu \mathrm{m}$. The pixel size is 1.2 arcsec in all bands, and two adjacent fields of view are imaged in pairs $(3.6$ and $5.8 \mu \mathrm{m} ; 4.5$ and $8.0 \mu \mathrm{m})$ using a dichroic beamsplitter. All four detector arrays in the camera are $256 \times 256$ pixels in size. The two short wavelength channels use InSb detector arrays and the two longer wavelength channels use Si:As IBC detectors, produced by Raytheon Infrared Operations ${ }^{2,3}$. The camera has an internal calibration subsystem that consists of a shutter mechanism that can close off the aperture to block external light, and calibration lamps that can illuminate the detectors through the IRAC optics to measure the system responsivity.

The IRAC instrument will address the four major scientific objectives defining the SIRTF mission ${ }^{4}$. These are (1) to study the early universe, (2) to search for and study brown dwarfs and superplanets, (3) to study ultraluminous galaxies and active galactic nuclei, and (4) to discover and study protoplanetary and planetary debris disks. IRAC is a powerful survey instrument because of its high sensitivity, large field, and simultaneous four-color imaging. In addition to the major goals cited above, IRAC is a general-purpose camera that can be used for a wide variety of astronomical investigations.

\subsection{Integration and Test sequence}

The IRAC instrument was assembled and tested at Goddard Space Flight Center (GSFC) as described previously ${ }^{1,5,6,7}$. In this paper we describe the calibration and optical performance testing performed at Ball Aerospace and Technology

\footnotetext{
* jhora@cfa.harvard.edu; phone 1617 496-7548; http://cfa-www.harvard.edu/irac; Harvard/Smithsonian Center for Astrophysics, 60 Garden Street, MS/65, Cambridge, MA 02138-1516
} 
Corporation (BATC) in Boulder, $\mathrm{CO}$ after the integration of the instrument with the Cryogenic Telescope Assembly (CTA). The IRAC cold assembly was mounted in the Multiple Instrument Chamber (MIC) ${ }^{8}$ in September 2000, and underwent a cooldown to $4 \mathrm{~K}$, warmup to room temperature, and cooldown again to $4 \mathrm{~K}$. The science instruments in the MIC have remained at liquid helium temperatures since then and will continue to do so until the cryogen is expended in flight. The IRAC Warm Electronics Assembly (WEA) was delivered to Ball in December 2000. The integrated assembly underwent EMI/EMC testing, vibration, and thermal vacuum testing in conditions approximating those to be encountered during and after launch. An end-to-end optical test was performed with IRAC, a module of the InfraRed Spectrograph (IRS) and the Pointing and Calibration Reference System (PCRS). After successfully completing these tests at BATC, the assembly was shipped to Lockheed in Sunnyvale, CA where it was integrated with the spacecraft $(\mathrm{S} / \mathrm{C})$. The system is currently undergoing final tests before being shipped to the launch site.

Several tests were done to confirm the interface between the S/C and IRAC. The final flight version of the IRAC software was uploaded into the instrument in June 2001 and has been used in all testing since then. A full set of software verification tests were performed, and all requirements were verified or identified as non-critical deviations that were documented or waivers obtained. All connections in the cables between the IRAC electronics and cold assembly were verified, and functional tests showed that both side A and B of the instrument were working without any major fault. Calibration tasks were performed, including measuring the nonlinearity of the arrays, electrical crosstalk, and other parameters such as "first frame effect" and the stability of the dark frames. Most of these tests were performed with the dewar at superfluid helium temperatures, allowing us to operate the arrays at the same temperature as will be used in flight. The results in general show that IRAC meets or exceeds all of its requirements, and should perform extremely well in-flight. A sample of some of the results are described in the sections below.

\subsection{IRAC Sensitivity}

The IRAC estimated point source sensitivity is given in Table 1 for the standard integration times available to SIRTF observers. The values given are the point source flux that would be detected at a 5 sigma level for the given frame times, excluding confusion and assuming a sky background level consistent with the north ecliptic pole. The SIRTF top-level requirements for channel 1 and 4 point source sensitivity in these units are 3 and $28 \mu \mathrm{Jy}$, respectively, for a $500 \mathrm{sec}$ integration (5 sigma detection). The other assumptions that went into this estimate are described in our previous paper, except that we have updated the estimate of the noise pixels based on refinements to the optical model developed by BATC of the in-flight system 9 .

Table 1. IRAC 5 sigma point source sensitivities $(\mu \mathrm{Jy})$

\begin{tabular}{|c|r|r|r|r|}
\hline $\begin{array}{c}\text { Frame Time } \\
(\mathbf{s e c})\end{array}$ & $\begin{array}{r}\text { Channel 1 } \\
(\mathbf{3 . 6} \boldsymbol{\mu m})\end{array}$ & $\begin{array}{r}\text { Channel 2 } \\
\mathbf{( 4 . 5} \boldsymbol{\mu m})\end{array}$ & $\begin{array}{r}\text { Channel 3 } \\
\mathbf{( 5 . 8} \boldsymbol{\mu m})\end{array}$ & $\begin{array}{r}\text { Channel 4 } \\
\mathbf{( 8 . 0} \boldsymbol{\mu m})\end{array}$ \\
\hline 2 & 295 & 347 & 680 & 494 \\
\hline 12 & 29 & 35 & 99 & 113 \\
\hline 30 & 11 & 16 & 50 & 68 \\
\hline 100 & 4.2 & 6.9 & 23 & 35 \\
\hline 200 & 2.5 & 4.5 & 16 & 25 \\
\hline
\end{tabular}

\section{OPTICAL TESTS}

One of the major accomplishments of the SIRTF testing at BATC was the end-to-end optical test that was undertaken to verify the optical performance of the assembled telescope and science instruments ${ }^{10}$. The optical performance results are described elsewhere ${ }^{9,11}$, here we provide details of the IRAC data acquisition and reduction used in that analysis. There were three basic types of data acquired during the optical tests: focus, phase retrieval, and jitter data. IRAC data in channels 1,2, and 3 were used for the analysis, although channel 3 was limited by the low $\left(<10^{-5}\right)$ transmission of the dewar window. There was no detectable window transmission in channel 4, so no optical measurements through the telescope could be made at $8 \mu \mathrm{m}$. 


\subsection{Focus and Phase Retrieval}

The focus measurements were obtained to determine the secondary setting that corresponds to best focus in flight, and to confirm the confocality of the instruments (as best as could be done given the test limitations). The phase retrieval measurements were conducted to measure the wavefront error of the system at various places in the IRAC fields, and to assist in diagnosing any unexpected aberrations in the images. The test was not a direct measure of the optical performance that will be achieved in-flight because of the double-pass configuration, the reference flat and dewar window (which are not in the optical path in flight), and gravity loading of the system. Therefore, another goal of the test was to verify the optical model of all of these components including the telescope, so that an accurate model of the in-flight telescope could be constructed to predict the performance that SIRTF will actually achieve.

The focus and phase retrieval data acquisition were similar, except that the focus data were taken typically near $( \pm 30 \mu \mathrm{m}$ of secondary mirror motion) of nominal focus, and the phase retrieval data were taken at focus settings as large as \pm 120 $\mu \mathrm{m}$ from nominal. The source of illumination was the Short Wavelength InfraRed (SWIR) lamp ${ }^{10}$ mounted near the SIRTF focal plane in the MIC which provided, after double-pass through the SIRTF optics, a pointlike source in the focal plane that could be positioned anywhere in the field using an articulated reference flat mounted above the telescope. For maximum signal-to-noise $(\mathrm{S} / \mathrm{N})$, the lamp was operated constantly at its highest safe operating temperature. Due to the window transmission and the temperature of the source, this meant that the peak of an in-focus source filled approximately $25 \%$ of full well at 2, 5, and 200 seconds for channel 1,2, and 3, respectively, with most of the light falling within $\sim 9$ pixels. For the extreme out-of-focus images taken in phase retrieval, the same light fell on roughly 1500 pixels. Therefore the $\mathrm{S} / \mathrm{N}$ was much lower even at the longest integration time of $200 \mathrm{sec}$, and more frames were collected and averaged. In addition to the source flux passing through the telescope optics, there was a significant amount of light that was reflecting and scattering off the inside surface of the dewar window. This created a background in the images that had to be subtracted before the images could be analyzed. Since the articulated flat was external to the dewar and the background was being created inside, the background remained constant as the point source was moved across the field. Therefore the background could easily be subtracted from the images (see below). In general, we took frames in channels 1,2 , and 3 simultaneously, although the source was in only channels $1 \& 3$ or channel 2. The off-source frames were used to measure the scattered light background which then could be subtracted off when the data were processed.

Once the data were obtained, the images were converted to FITS format and transferred to a local workstation for processing. The goal of the processing was to remove all "instrumental signatures" such as detector non-linearity, pixelto-pixel gain differences, etc., in order to compare the observed images to simulated images based on the telescope and test setup model. The processing steps were as follows: first, a previously obtained dark frame was subtracted from each image. The dark appearance is different for each array, and is dependent on the Fowler number and frame time being used, so the frame has to be matched to a dark frame with the same parameters. The linearity correction was then applied, using a fit that was performed independently on each pixel of the image. At this point the channel 3 output columns were normalized to remove constant offsets that occur between them within an image that produce a "striping" effect (see Figure 1).

Additional steps were then performed in order to subtract the background. First, for every set of frames in the same channel and integration time, the frame medians were normalized to remove any offset variations. Then a background frame was constructed from a median of the frames taken when the point source was in the other field of view. This step reduced the noise in the background frame and eliminated cosmic rays and other transient effects from the background. Once a background frame was made for each channel and frame time, the background was subtracted and the pixel gain correction applied. The gain correction was determined from tests at GSFC, and included both pixel-to-pixel gain differences and larger spatial scale effects due to optical transmission of the system at different field points. Finally, the images were flipped and/or rotated to remove all optical and electronics transformations in order to put the images from all channels on the same coordinate system to make it easier to compare to the simulated images and reduce confusion.

A result of this reduction is shown in Figure 1. The data were taken at a secondary mechanism position of $-120 \mu \mathrm{m}$ relative to nominal focus with a $200 \mathrm{sec}$ frame time in channel 3. This is the farthest excursion from nominal focus used, and channel 3 has the lowest signal, so this is the worst $\mathrm{S} / \mathrm{N}$ case in the dataset. The background subtraction and column 
offset correction have the largest visual effects, the detector dark frame is fairly uniform and the gain correction is only at a level of a few percent. Also shown is a reduced image in channel 1 near best focus.
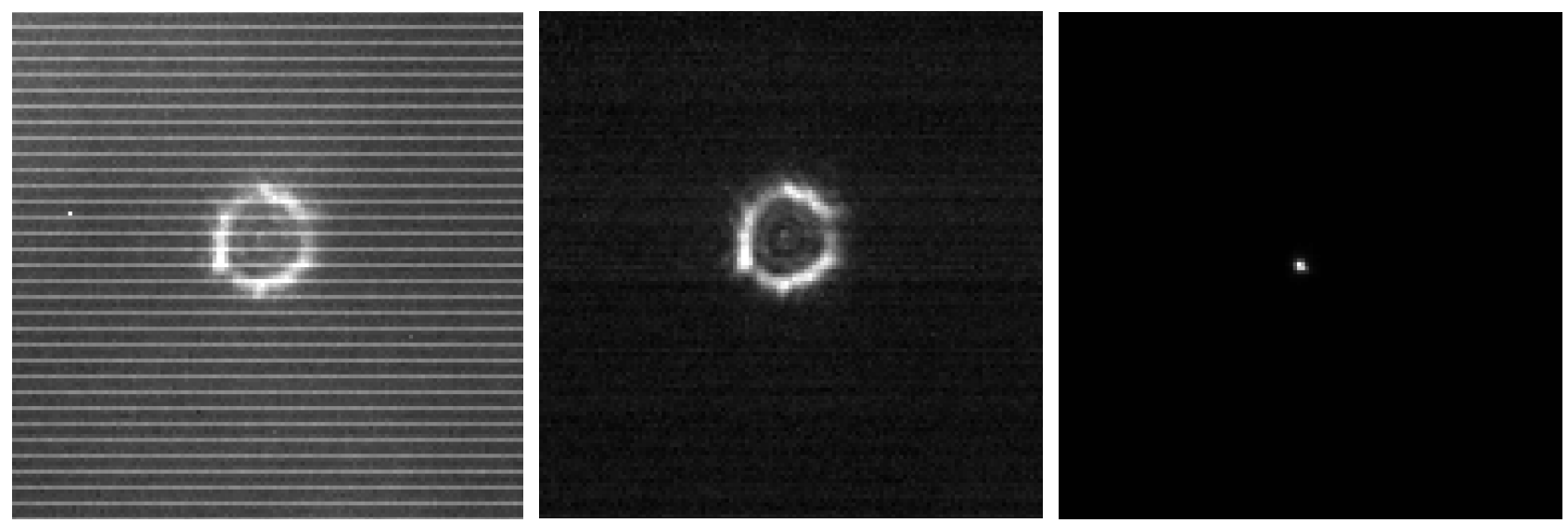

Figure 1. IRAC optical test data, showing the central 128x128 pixels. The image on the left is a raw 200 sec channel 3 image, -120 $\mu \mathrm{m}$ secondary position (relative to best focus. The center one is the result of the left image after the reduction pipeline. The image on the right is channel 1 , near the best focus position.

The results of the focus determination and optical quality measurements are summarized elsewhere in these proceedings ${ }^{9,11}$, but the basic result is that the SIRTF/IRAC optical system is performing better than required to be diffraction-limited at $6.5 \mu \mathrm{m}$. Based on the CodeV models of the telescope and IRAC developed at Ball and GSFC, we can predict the in-flight properties of the images in the IRAC focal plane. These are summarized in Table 2. The estimate assumes that the telescope focus is set to the best IRAC overall focus, which is a compromise between the best focus settings of the individual channels.

Table 2. Predicted in-flight IRAC image quality properties

\begin{tabular}{|c|c|c|c|}
\hline Channel & Noise pixels & $\begin{array}{c}\text { FWHM } \\
(\operatorname{arcsec})\end{array}$ & $\begin{array}{c}\text { Central pixel } \\
\text { flux (\%) }\end{array}$ \\
\hline 1 & 10.5 & 1.3 & 46 \\
\hline 2 & 13.0 & 1.3 & 38 \\
\hline 3 & 14.3 & 1.6 & 32 \\
\hline 4 & 18.6 & 1.7 & 21 \\
\hline
\end{tabular}

Pointing jitter was modeled as a Gaussian blur of radius 0.3 arcsec, and charge diffusion of 0.486 pixels in channels 1 and 2 only. The "noise pixels" column gives the equivalent number of pixels whose noise contributes to noise in the analysis when an image is spatially filtered for optimum faint point-source detection. The noise pixel numbers were calculated from the normalized PSF pixels of intensity $P_{i}$ at each pixel $i$ using the relation below:

$$
\text { NoisePixels }=\left(\sum_{i} P_{i}^{2}\right)^{-1}
$$

where $\sum P_{i}=1$. For the FWHM and central pixel flux, no charge diffusion or jitter were added, so these are the lowest FWHM or highest central pixel flux possible based on the predictions.

\subsection{Jitter measurements during optical tests at Ball}

The optical test setup at BATC was designed to be stable and minimize random image motions or jitter in order to be able to integrate up to hundreds of seconds required to achieve adequate $\mathrm{S} / \mathrm{N}$ in the tests. We obtained jitter 
measurements in order to confirm this and to characterize the actual observed jitter to include this parameter in our simulated images that were compared to the observed focus and phase retrieval data.

To perform this measurement, we obtained data in channel 1 in subarray mode at several frame times. In subarray mode, a $32 \times 32$ pixel subsection of the array is read out with the same pixel sample time used in full-array mode, providing frame times down to $0.02 \mathrm{sec}$. Given the intensity of the source in channel 1, a frame time of $0.1 \mathrm{sec}$ was necessary to achieve a S/N of $>100$ in each image, which was the minimum useful frame time. The data were reduced similar to the method used for the full array data, described above in Section 2.1. The jitter measurement was done before or after each of the major focus or phase retrieval tests performed. An example is shown in Figure 2.

\section{1/10/10 Jitter 0.1 sec subarray}

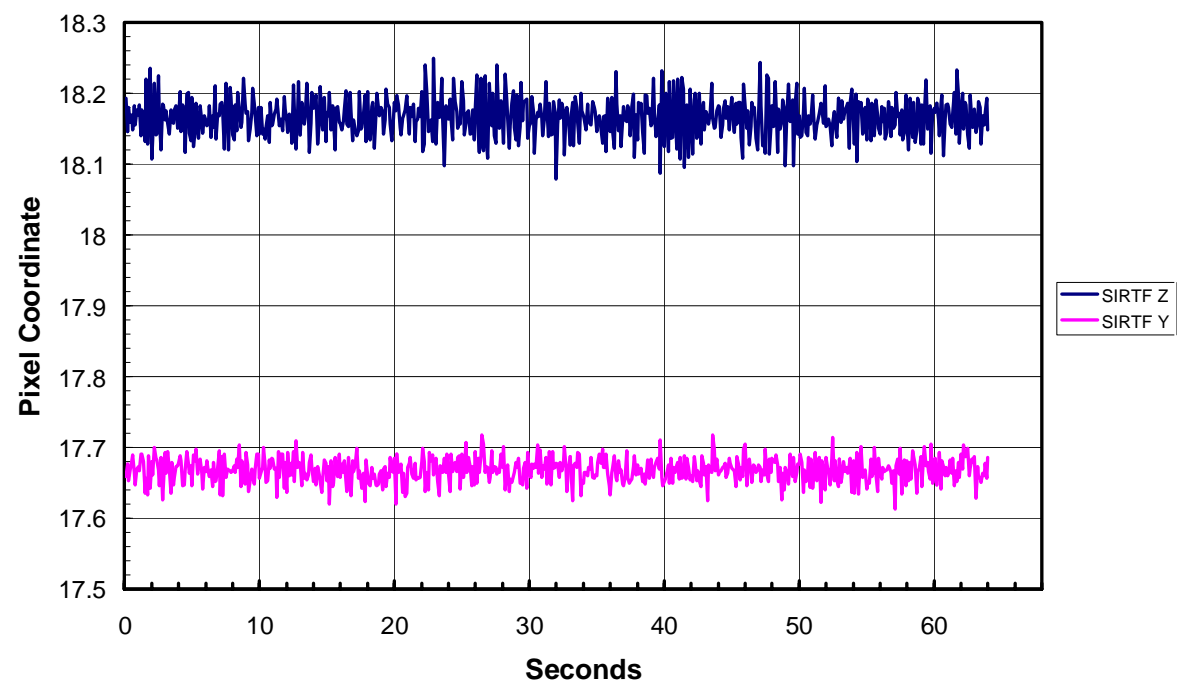

Figure 2. IRAC Jitter measurements during optical tests at BATC. The pixel coordinates of the centroid are plotted as a function of seconds during the measurement. The $\mathrm{Z}$ axis is the top trace, the $\mathrm{Y}$ axis is the bottom one.

The standard deviations for the result shown are approximately 0.03 and 0.02 pixels for $\mathrm{Z}$ and $\mathrm{Y}$, respectively. The value for the $\mathrm{Y}$ axis jitter is roughly what one would expect to see for the errors in the centroid calculation caused by the image noise at the observed $\mathrm{S} / \mathrm{N}$ level. The $\mathrm{Z}$ axis shows a higher level and is probably detecting some real jitter. The maximum deviations from the average centroid are $<0.1$ pixel, and there is no detectable drift over the period of the measurement. Therefore the images during the optical tests were very stable and image blur due to jitter was a negligible effect. A variation of this technique will be used to evaluate jitter once in flight, to assess its effects during the telescope focus operation.

\section{INSTRUMENT CALIBRATION TESTS}

Many calibration tests were performed at the IRAC subsystem level previous to its delivery to BATC, including characterization of the arrays ${ }^{11,13}$ and IRAC optics ${ }^{14}$. However, at GSFC we did not have the flight cables available which limited the applicability of some of the tests. In addition, some slight changes to the array biases were made that invalidated some of the previous calibrations and so additional measurements were performed at BATC.

\subsection{Linearity}

The detectors have a non-linear response to photons over the range of zero to full-well. The goal of the linearization task is to correct the measured output of the detectors such that there is a purely linear relationship in the image between photons falling on the detector and the digital numbers (DN) in the processed images. Errors in this correction will 
directly affect IRAC's photometric accuracy, both for sources of different brightness in a single field, and for comparing measurements of sources taken with different exposure times.

Measurements of the system nonlinearity were made by illuminating the focal planes with a lamp of constant intensity, and changing the exposure time to fill the detector wells to varying degree. Frames with the lamps off were also obtained in the same way as the lamp-on data in order to subtract the detector dark frames to determine the measured DN for each exposure. The linearity correction can be done in any number of ways. For the InSb detectors of channels 1 and 2 , a relation of the form

$$
S=A \cdot t+B \cdot t^{2}
$$

(where $\mathrm{t}$ is exposure time and A and B are constants) was used to fit to the measured DN values. The linearized signal $S^{\prime}$ is then given by

$$
S^{\prime}=A \cdot\left(\frac{-A+\sqrt{A^{2}+4 \cdot B \cdot S}}{2 \cdot B}\right)
$$

Figure 3 shows an example of the result of this fit for channel 2 data, where the relative errors between this function and the data are shown. The linearity correction performs well over the range, with deviations from the model of $\sim 0.5 \%$ peak-to-peak, up to about $90 \%$ full-well. The data are stable to a couple tenths of a percent. The curve on the left bends sharply downward past 40,000 DN where detector saturation occurs. Similar data were taken for the other IRAC channels, and will be used to determine the nonlinearity correction parameters. The non-linearity correction will be applied in the data pipeline by the SIRTF Science Center (SSC) before delivery to the observers.
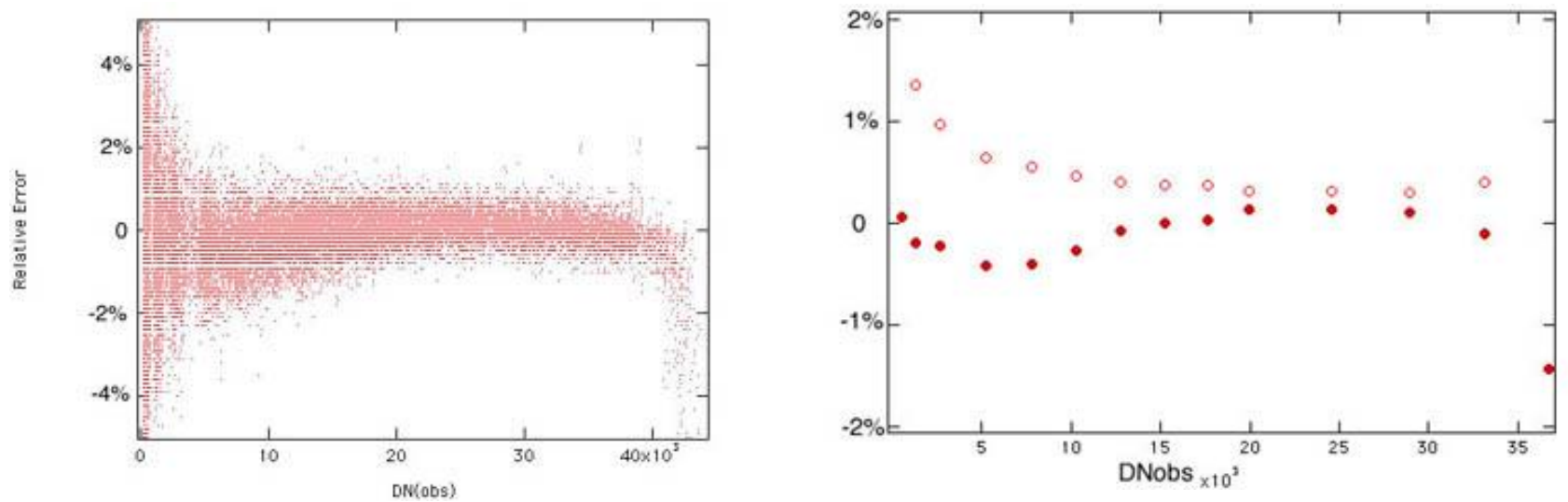

Figure 3. Relative pixel error in the linearity determination for channel 2. The measured DN is on the horizontal axis, and the vertical axis shows the relative error between the measured DN and that expected by the linearity correction fit, in percent of DN. The left plot shows all pixels, the right plot shows the relative error binned by input image. Filled circles are the mean fit error, and the open circles are the standard deviation around the mean.

\subsection{Electrical Crosstalk}

Since the detectors in all four channels are usually read out simultaneously, a potential exists for crosstalk. The output lines run from the cold assembly to the WEA through many connectors and cables, and the analog electronics are physically in the same box with the rest of the IRAC electronics. If the lines are not properly shielded along this path, signal from one channel could possibly get into another and create a false source. Channels 1 and 3 view a different field than channels 2 and 4, and the orientation on the sky of the channels that view the same field are flipped, so in general the crosstalk will appear at a different array location than the bright source in the other channel. Since all the cables and connections between the IRAC warm electronics and cold assembly changed when we were integrated with the CTA, a test for crosstalk needed to be performed at this stage. The test was limited in that we could not put bright point sources on channels 3 and 4, but we could adequately check for crosstalk for sources in channels 1 and 2. 
Crosstalk tests were performed in August 2001 at BATC. In these tests, one channel was illuminated by the SWIR source, and all four channels were read out simultaneously. A set of 50 frames were obtained from each channel. Then the illuminated channel was turned off, and another set of frames were obtained in the three channels not directly illuminated. Each set of frames from a channel were averaged to improve S/N. The averaged frame "pairs" (illuminated channel on and illuminated channel off) for each unilluminated channel were subtracted to look for evidence of any residual ("ghost") image in the same pixel location as the source image in the illuminated channel. This was done for two separate locations on channels 1 and 2.

The results were that the electrical crosstalk was extremely low (a maximum of $0.01 \%$ in one case) or not detectable at greater than the $0.002 \%$ level. To put this in context, for a saturating source in the illuminated channel, the worst case crosstalk will be less than the sky background in the lowest background channel and shortest standard frame time.

\subsection{Stray and scattered light}

When IRAC was integrated into the MIC, it was realized via visual inspection that the channel between the pickoff mirrors and parts of the IRAC alignment fiducial were acting as reflecting surfaces that could potentially illuminate the MIC (directly and from reflection from the secondary). The channel was painted black and a cover installed over the fiducial so that these surfaces would not create a stray light problem. A series of tests were performed where the SWIR source was placed on these locations and others to confirm that those issues had been solved.

However, during those tests, when the SWIR source was moved off the array to obtain a background frame, a glint was seen in channel 2 when the point source was in a specific location (pixel coordinate 17,-53) off the field (see Figure 4). The peak brightness of the glint was approximately $0.14 \%$ of the peak of the SWIR source, and the glint was present for SWIR source positions in a 30 arcsec region off the lower left of the array. The glint changes its appearance slightly as the source is moved, changing position and intensity. It is likely that the glint is caused by a reflection from the ceramic board that the array is mounted on, or some component on that board. The pickoff mirror which is the defining field aperture is slightly oversized, so that a region around the array is illuminated. The glint was also observed in channel 1 , and is expected to be present in channels 3 and 4 . Observers should avoid placing bright sources in that off-field location, and should be aware that sources that are in that position can create glints of this type.

Another feature that was seen is shown in the image on the right of Figure 4. The source is at location $(-35,-35)$ pixels, and a small amount of diffuse scattered light is seen on the array, with the peak intensity being about $0.006 \%$ of the brightness of the peak which is off the array. Since this is such a low intensity feature, it will only cause a detectable background increase for saturating sources in that location off of the array. The glint described above and the slight increase in scattered light shown in the right image of Figure 4 are the only significant scattered light features observed during the system tests at Ball.
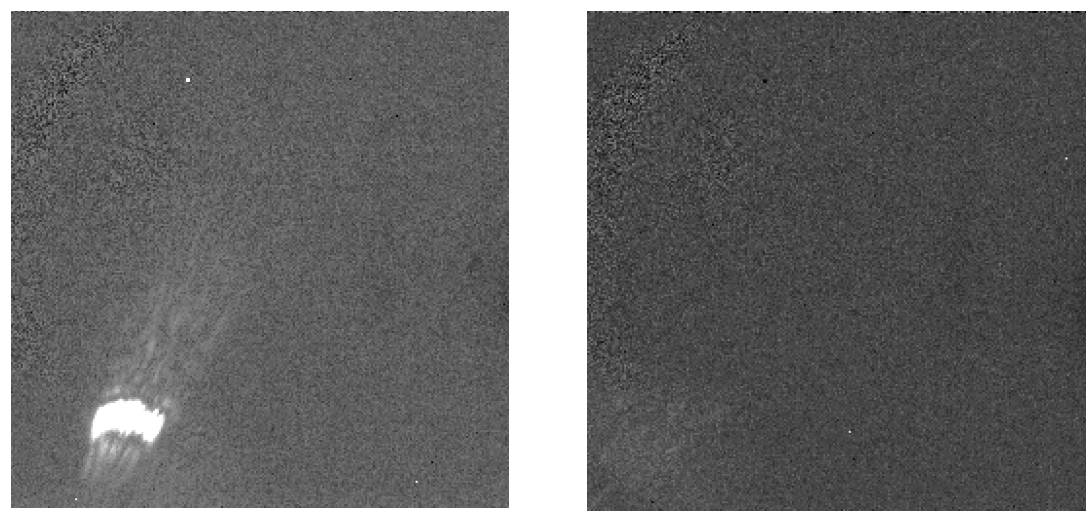

Figure 4. Scattered light features in channel 2. On the left is shown the brightest of the features found, the peak of which is $0.14 \%$ of the peak brightness of the actual source (which is off the array to the lower left). On the right is shown another faint feature in the lower left corner, at approximately $0.006 \%$ of the peak of the original source. 


\subsection{WEA temperature characterization}

The purpose of the test was to determine the changes in instrument responsivity and dark frame characteristics as a function of WEA temperature. The IRAC WEA is mounted on the spacecraft and is not actively temperature controlled, although there are heaters that will keep it above its survival temperature. Thermal models have predicted that IRAC will operate in the range sampled during this test, and that the temperature will be stable to $\pm 5{ }^{\circ} \mathrm{C}$ during the periods that IRAC is on. The same set of measurements were performed at four different WEA baseplate temperatures: $-5,+6,+17$, and $+28{ }^{\circ} \mathrm{C}$. The baseplate was controlled to this temperature, and when the temperature of one of the internal WEA cards (FPE1ATMP) had stabilized so that it was changing less than one degree C per hour, the measurements were started. This board was typically about 15 degrees warmer than the baseplate.

The following measurements were performed at each temperature: dark frames (shutter closed), transmission calibrator frames, flood calibrator frames, and observations of the SWIR source in the MIC. The arrays were maintained at a constant temperature ( $15 \mathrm{~K}$ for channels 1 and $2,6 \mathrm{~K}$ for channels 3 and 4 ) so the appearance of the dark frames remained relatively constant at all temperatures sampled in the test. The median level of the dark frames had a slight temperature dependence in some channels, up to $0.3 \mathrm{DN} /{ }^{\circ} \mathrm{C}$. This is a relatively small change given the expected stability of the WEA and the changing backgrounds on the sky encountered when moving to different objects.

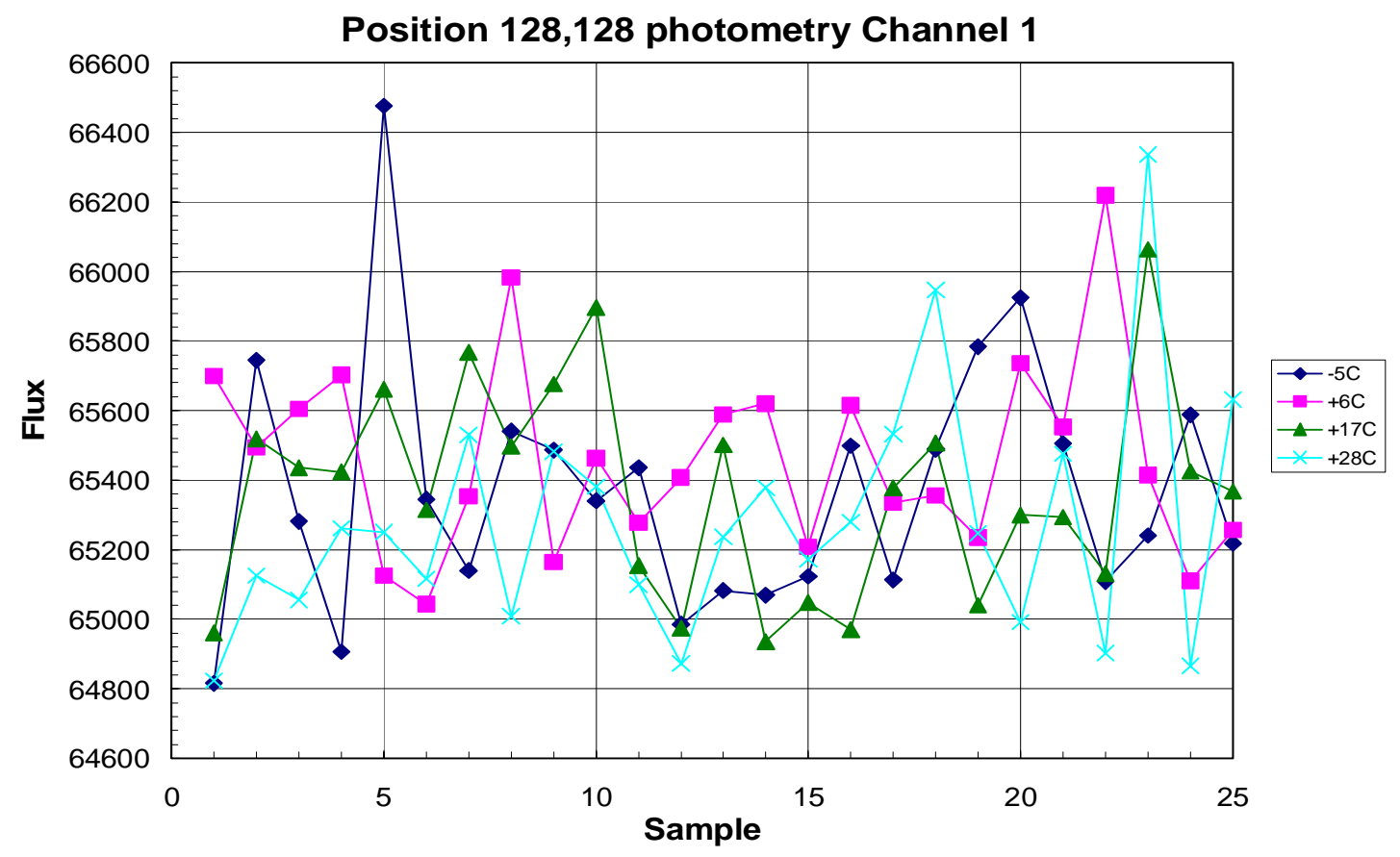

Figure 5. Photometry of the SWIR source during WEA temperature characterization test. The source was located near the center of channel 1 for all temperatures. The source flux was extracted from individual images using the DAOPHOT package of iraf. Each trace shows samples at a different WEA temperature, as labeled.

The internal calibration lamps (transmission calibrators, flood lamps) were also monitored at the changing WEA temperatures. The lamps are intended to operate at the same current whenever they are powered on, set by resistors and the voltage applied in the WEA, so they could be affected by changes in temperature. However, the only way to measure the lamp brightness is with the IRAC arrays, therefore changes in the array responsivity cannot be separated from changes in the lamp for any single measurement. 
The measurements of the transmission calibrator lamps showed a very stable result across all temperatures, with no clear trends. The lamp changes are $<1 \%$ over the full range, and a change of $\leq 0.03 \% /{ }^{\circ} \mathrm{C}$ over the range sampled. The flood lamps, however, each showed a more or less monotonic change with WEA temperature, most getting fainter as the temperature increased. The maximum change was approximately $0.03 \% /{ }^{\circ} \mathrm{C}$.

The SWIR source was also observed in channels 1 and 2. The power supply of the SWIR source remained at room temperature throughout the test, so it should have been stable throughout the test. The source was placed in five positions on the array and roughly 25 samples taken at each location. Photometry of the source was performed on each image. The result for channel 1 is shown in Figure 5. There is no clear trend with temperature, the variations between the average photometry at each temperature are approximately $0.15 \%$. Channel 2 showed a similar result. Since both the transmission lamps and the SWIR source measurements seem constant, we conclude that the response of the arrays themselves are mostly independent of the WEA temperature. The largest effects of changing WEA temperature seem to be an offset level shift of the darks, and some small variations of the flood lamp intensity.

\subsection{Dependence of frame median on previous frames ("first frame" effect)}

The IRAC shutter will be open in flight, allowing light to constantly fall on the array, even during slews and downlink. When IRAC is not currently taking an exposure, the FPA electronics reset the array every $0.2 \mathrm{sec}$ to avoid having an excess charge build up on the array. If frames are being taken back-to-back, there is only a single reset between frames and the dark frame has a slightly different appearance for the first frame compared to the subsequent ones, in addition to the shift in offset level. For this reason, it was decided that in flight all frames would be "first frames", i.e., taken with a delay time between frames. In practice this is not inefficient since one is usually moving the telescope between frames so the delay will occur as the telescope settles on the new position. Also, when changing observing parameters such as

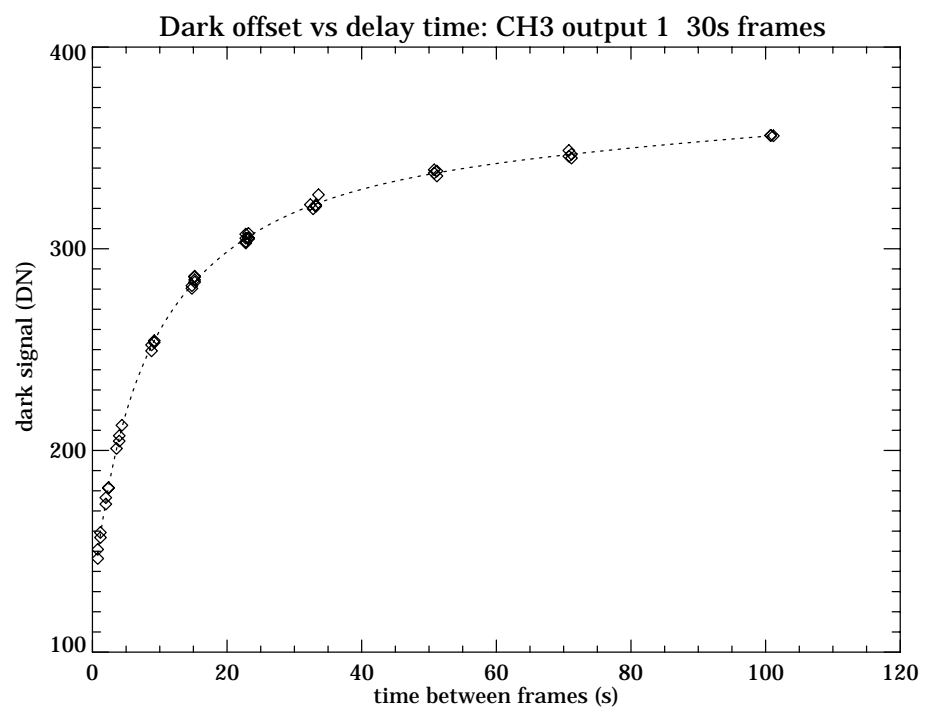

Figure 6. Dark offset (median of dark image) as a function of delay times between frames in channel 3,30 sec frames. frame time or Fowler number, a new command needs to be sent from the spacecraft. Therefore even frames done at the same telescope pointing have short delays between them that result in multiple resets.

However, it was found that the appearance of dark frames in all 4 IRAC channels are not constant, even with multiple resets between them. A dark image taken with a particular frame time and Fowler number depends on the amount of time lapsed since the previous image, and the frame time and Fowler number of the previous image. In a sequence of images of the same frame time and Fowler number, the variation from one image to the next consists principally of a uniform change over the whole image, and to a much smaller extent, a change in the relative offsets on each of the 4 outputs of an array, which appears as "pin striping" (see Figure 1), and, finally, to small spatial gradient across the image. Channel 3 has by far the largest variations in darks. In the extreme case of a short integration, followed after a long delay by a $200 \mathrm{sec}$ frame which is repeated several times with no delay, the difference between the first and last $200 \mathrm{sec}$ dark can be over 1300 e-. It is unlikely that such a sequence of images would ever be taken on SIRTF. Generally, one will be concerned with dark variations in a sequence of images of the same kind, where the time between images may vary from $\sim 8 \mathrm{sec}$ to $\sim 60 \mathrm{sec}$. An example of the dependence of the dark offset on the time between frames is shown in Figure 6, which shows the results of a calibration of the standard $30 \mathrm{sec}$ darks. A sequence of 48 images were taken, and the time between frames ranged between 0.8 and $101 \mathrm{sec}$. The mean of the pixels near the center of channel 3, from output 1, are plotted. The first 
image in the set is not plotted, because its offset was different since it was not preceded by a $30 \mathrm{sec}$ frame. The dark offset increases monotonically with the time between frames. The dotted curve is a fitted function which is the sum of a constant and 3 exponential terms.

The IRAC dark frames were calibrated in tests at Lockheed at flight-like array temperatures and the shutter closed. The dependence of the dark image on the time between frames of the same type was calibrated for all the standard frames for delay times from less than $1 \mathrm{sec}$ to $60 \mathrm{sec}$ (for short frames) or $101 \mathrm{sec}$ (for long frames). The HDR mode darks were also calibrated over a range of delay times. Channel 1 has very significant variations in offset, particularly in light of the low zodiacal background in that band expected in-flight. Channel 2 has very small variations in offset, and channel 4 has significant variations, though they are small compared to the expected in-flight background. Also, in general, the variations in all channels are much smaller for short frame integrations than for long integrations.

The first image in an AOR usually would not be covered by the dark calibrations described above, because it is likely to be preceded by a frame of a different kind. For such cases, as for example, going from a $12 \mathrm{sec}$ frame to a $100 \mathrm{sec}$ frame, there is only a sparsely sampled set of data. Therefore it is unlikely that, at the beginning of the mission, we will be able to provide a dark correction for the first image in an AOR.

\subsection{Latent images}

All the IRAC channels have latent, or residual, images of a source after it has been moved off a pixel. When a pixel is illuminated, a small fraction of the photo-generated charge is trapped. The traps have characteristic decay rates, and can release a hole or electron which will accumulate on the integrating node long after the illumination has ceased. In all arrays, the longest e-folding decay time is about $1000 \mathrm{sec}$. In a sequence of images that begins just after a star has been

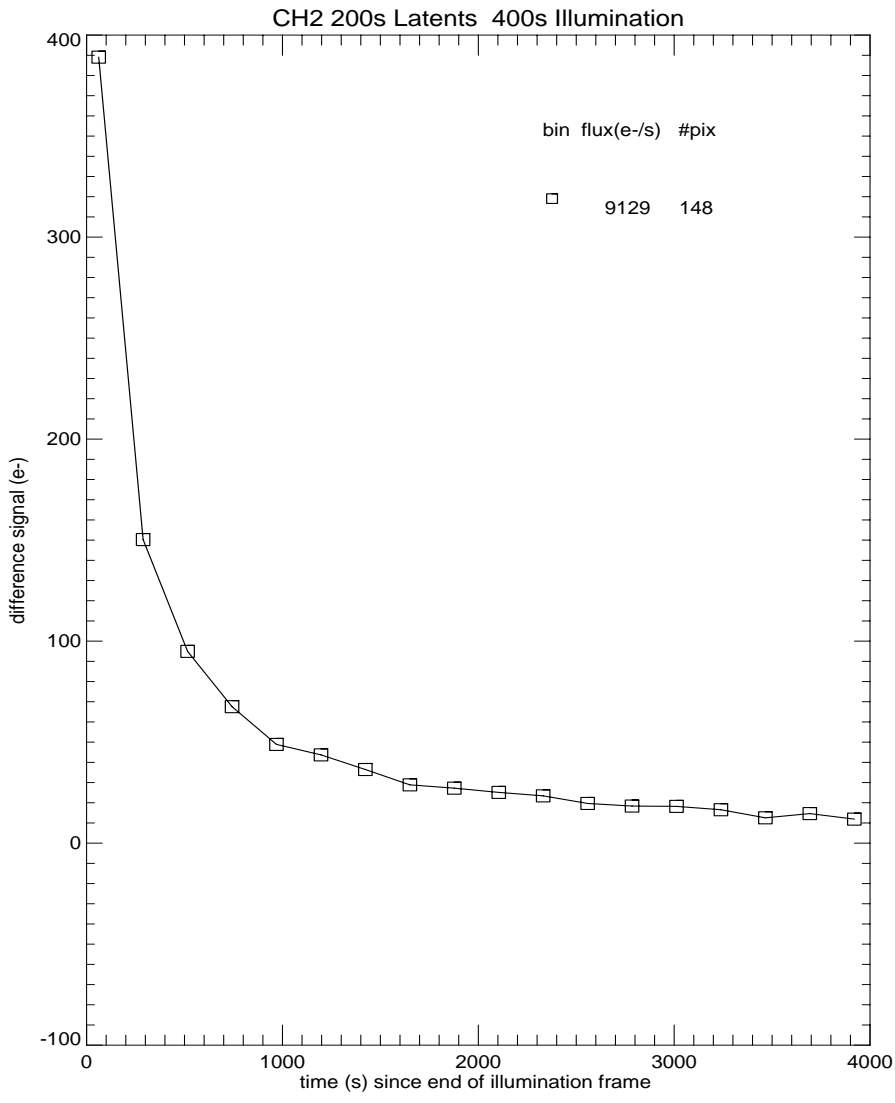

Figure 7. Latent image in $200 \mathrm{sec}$ channel 2 frames. moved off a pixel, the latent fluence in the pixel drops rapidly at first and slowly later. Even in a long integration that begins shortly after the illumination ends, the latent fluence never exceeds $1 \%$ of the fluence when the pixel is illuminated. Usually the latent fluence will be orders of magnitude smaller, but the latent image of a star can persist as a false point source for tens of minutes after. In channel 1 , with a low background, it takes $\sim 15$ minutes for a latent image of a bright source to fall below the 3-sigma point source detection threshold in $200 \mathrm{sec}$ images. The time to decay below the threshold decreases with longer wavelength, and in channel 4 it relatively short, because of the relatively high background.

The latent effect was calibrated in October and November 2001 at BATC using the SWIR, the Long Wavelength IR source (LWIR), and the internal transmission calibrator (TCAL) lamps. The calibration was completed with the TCAL in April 2002 at LMMS. The SWIR was used to verify that latents from a point source behave the same way as latents from a diffuse source. With the LWIR and combinations of the TCAL lamps, nearly every pixel in each array was sampled at low, medium, and saturating fluxes for various integration times. The rate at which traps are populated for a given flux depends on the voltage across the detector diode, which decreases as fluence increases; in InSb the rate increases as a pixel discharges, but in Si:As the rate 
decreases. To be able to predict the latent fluence in a pixel following exposure to an arbitrary flux requires that we know the trapping efficiency and the number of traps at or near trap saturation, and the product of the efficiency and the number of traps at fluences well below trap saturation. Trap saturation occurs when the flux is so high that nearly all the traps are populated; this occurs, very roughly, for fluxes greater than about $300000 \mathrm{e}-/ \mathrm{s}$. Unfortunately, no available source was nearly this bright, so the latent calibration for very bright sources will have to be done on stars.

Figure 7 shows an example of a sequence of $200 \mathrm{sec}$ frames in channel 2 following a $400 \mathrm{sec}$ integration on the LWIR source. The dark-corrected average fluence is plotted for the mean of 148 pixels in the brightest part of the illumination, where the flux was $9129 \mathrm{e}-/ \mathrm{s}$. The pixel wells were very strongly saturated. The abscissa is the time of the beginning of the $200 \mathrm{sec}$ frame, measured from the moment the lamp was turned off. Even after almost $4000 \mathrm{sec}$, the latent image is still visible, though it is well below the noise per pixel with a low background in flight, which is about $40 \mathrm{e}-\mathrm{rms}$ in a 200 $\mathrm{sec}$ frame. Latent images were measured with a range of integration times, from $2 \mathrm{sec}$ to $200 \mathrm{sec}$, to sample the fast and slow decays. The latents are fitted very closely by a model with 5 "species" of traps, which have distinct exponential decay times (in InSb) of about $0.7,5,30,180$, and $1000 \mathrm{sec}$. The latents are about the same for most pixels. A small part of the channel 2 array has somewhat different latent characteristics.

In the early part of the mission, each pixel will be flagged when there is potentially a significant latent fluence in it. The flagging will be done with a model that uses the mean characteristics per array. We expect to provide, along with each $\mathrm{BCD}$, and not too long into the mission, an image with an accurate estimate of the latent fluence in each pixel, based upon the fluences in preceding images and the measured characteristics of each pixel.

\section{IRAC SHUTTER AND INSTRUMENT STABILITY TESTS}

\subsection{IRAC shutter anomaly}

IRAC's shutter performs two main functions - it closes the instrument off from external light in order to obtain dark frames, and it has a mirror surface on the internal side that is used to view an integrating sphere system with two calibration lamps that can be turned on to provide a responsivity and relative response calibration. Because of the potential for it to permanently block the aperture, the mechanism was designed such that if power was removed or any other electrical fault occurred in the wiring or drive electronics, a mechanical spring would return the shutter to the "open" position. Also, the shutter is redundant with two separate drive coils so that it can be operated from either "side A" or "side B" of the IRAC warm electronics.

In mid-May 2001 during a routine functional test, the shutter was closed on side A and failed to open when commanded, and stayed closed when the power to the instrument was removed.. This anomaly occurred after a change in the flight software subroutine that operated the shutter. During troubleshooting following the anomaly, the shutter opened, but it was found that it could be made to stay closed on side A with the power off under certain conditions. The shutter could not be made to stay closed after removal of power on side B. Subsequent tests on the flight system and extensive testing with engineering models and flight spares at GSFC determined that a residual magnetism develops in the mechanism between the rotor and stator contact points that is strong enough to resist the spring force and keep the shutter from opening. The magnetism on side A was stronger than side B because its mechanism was more accurately aligned and the rotor and stator came into closer contact. It was determined that a slight current reversal was necessary to reliably operate the shutter by canceling out the residual magnetism and allowing the spring to open the shutter. A slight change in the flight software to automatically apply the reverse current was developed, and this was tested and proved to function without fault for many cycles.

However, it was decided by the SIRTF Project Office that due to perceived risk and schedule concerns, it would not allow the shutter to be operated in flight. Without the shutter, IRAC cannot obtain dark and responsivity calibration frames quickly, and the ability to perform an absolute measurement of the background is lost. We also had to develop a strategy on how to calibrate the instrument without the shutter. Of immediate concern were the dark frame and instrument response stability, and any calibration observations that would be difficult to obtain in-flight without the transmission calibrator. Our nominal calibration plan which included the shutter was to close it at regular intervals of 6 
hours or less to perform a quick measurement of the detector dark frame, and also to take transmission calibration frames to monitor changes in the pixel-to-pixel relative response.

A calibration strategy is being developed for derivation of the IRAC calibration in-flight using celestial sources such as the zodiacal background. This strategy will be tested and refined in flight, and we hope to achieve a performance level similar to that originally designed for IRAC. The most immediate impact will be an inability to accurately measure the absolute background level.

\subsection{Three-day Stability Test}

Given that the shutter was not to be used in flight, the calibration plan had to be changed to not involve the internal calibration lamps or use of the shutter for dark frame observations. Since detector background frames will now take several hours of observations on the sky as compared to minutes with the shutter, there is a strong incentive to maximize the time between calibration measurements. In order to determine the stability of IRAC over longer periods of time typical for the in-flight operation, a 3-day stability test was performed at BATC. IRAC was powered on almost continuously over a three-day period, and thousands of frames were obtained.

\subsubsection{Zero point frames}

One of the primary uncertainties before the test was the stability of the dark frames over long periods. A sequence of darks with the standard frame times were obtained throughout the entire test at regular intervals. Figure 8 shows some sample results for channel 1. The median DN levels are plotted in Figure 8 as a function of time during the test. The same sequence of frames were repeated each day of the test. One can see roughly the same variation pattern of the darks in each of the days. The short frame time darks showed very little change throughout the test. The longer frame times show a decay in the DN level over time in each set of frames, which may be in part the decay of latent images induced when the arrays are exposed to flux in the frames immediately preceding the darks.

\subsubsection{Sample Observations}

During the three-day stability test a numbers of simulated Astronomical Observing Requests (AORs) were performed that are similar to those that will be executed in flight. One of the simulated AORs utilized high dynamic range observations, specifically, a series of alternating 0.6 and $12 \mathrm{sec}$ exposures, with 10 second delays between each pair where a dither would take place in flight. There were eight repetitions of this AOR, generally separated by a couple hours, with some longer 12-hour timescale gaps. The lamps were off during this time, so these are dark frames and different illumination levels between the frames are not an issue. After the data were collected, processing was performed with the SSC pipeline "pre-processor", which corrects for the InSb sign flipping, the wraparound effect (from bit truncation), and the Fowler-sampling/barrel-shifting. The median of the pixels was calculated from a 50x50 box in the center of each array.

The medians plotted as a function of time are shown in Figure 9. The mean dark frame DN values with short frame times are very stable. Their scatter over the whole test is $0.3 \mathrm{DN}$, or about one electron. For the 12-second frames there is obviously something more complex happening. Intuitively, one would have expected the first long and short frames to have been different, owing to the first-frame effect (see §3.5). Since the timing is always the same on the following exposures, one would have expected them to have nearly the same values all the time. The data reduction plan was to correct for the first frame effect, and then subtract the same pair of offsets from each HDR pair. We do actually see roughly two offsets for each exposure time. However, we see above that the first run after a long hiatus has a different offset than consecutive runs (this occurs after the 3rd and 6th run). We also see what looks like a drift downwards within a run and a slight drift upwards between all the runs. However, the simulated observations are in general extremely stable. The difference is about $2 \mathrm{DN}$. If a constant value was used all the time for the $12 \mathrm{sec}$ frames, it would result in a 1 sigma scatter of $1.1 \mathrm{DN}$. 


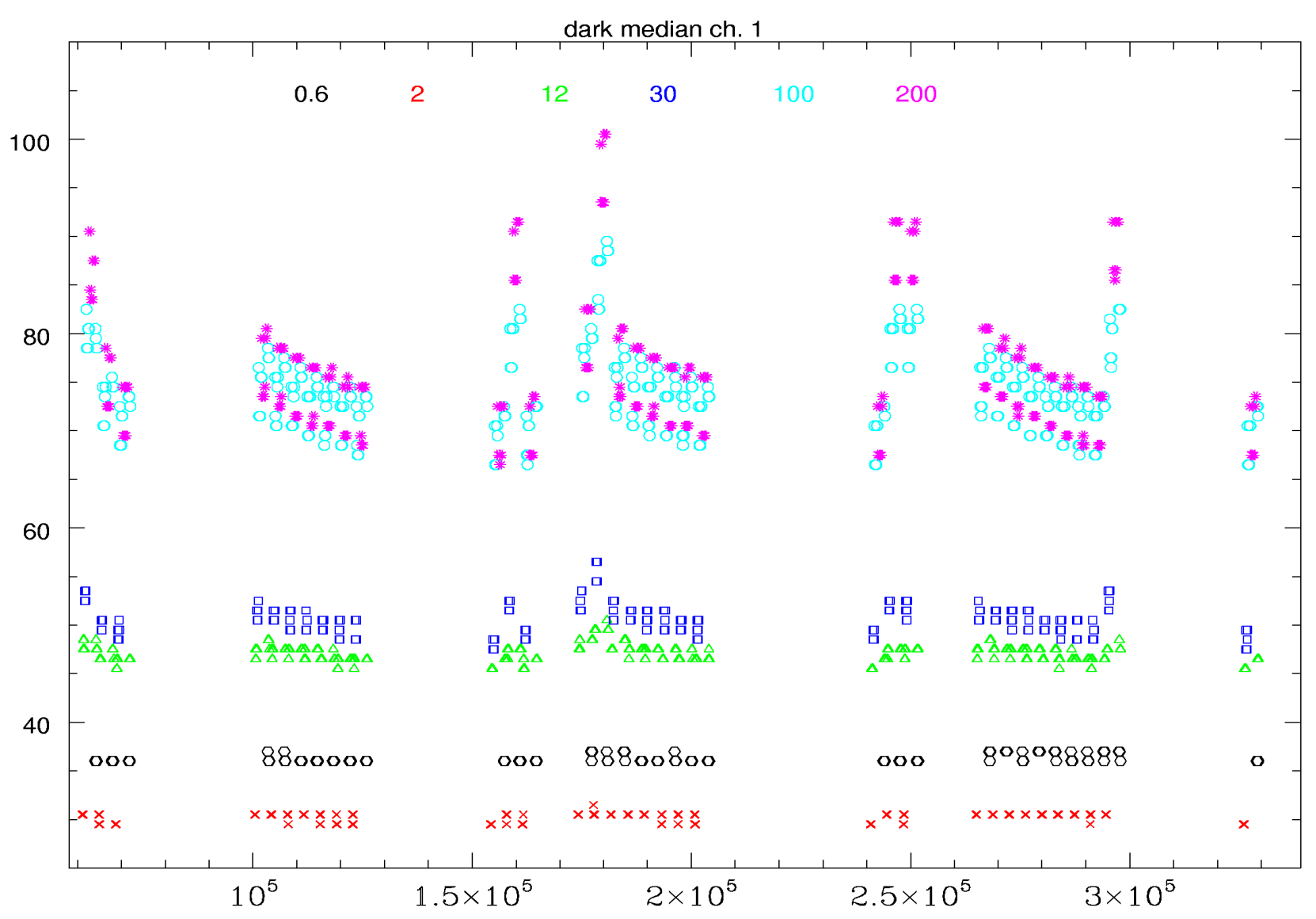

Figure 8. Median levels of dark frames throughout the 3-day stability test. Median counts in each dark frame are plotted as a function of elapsed time. Different integration times are represented by different colors and symbols, listed at the top. The horizontal axis is in units of seconds since the test start, the vertical axis is in DN.

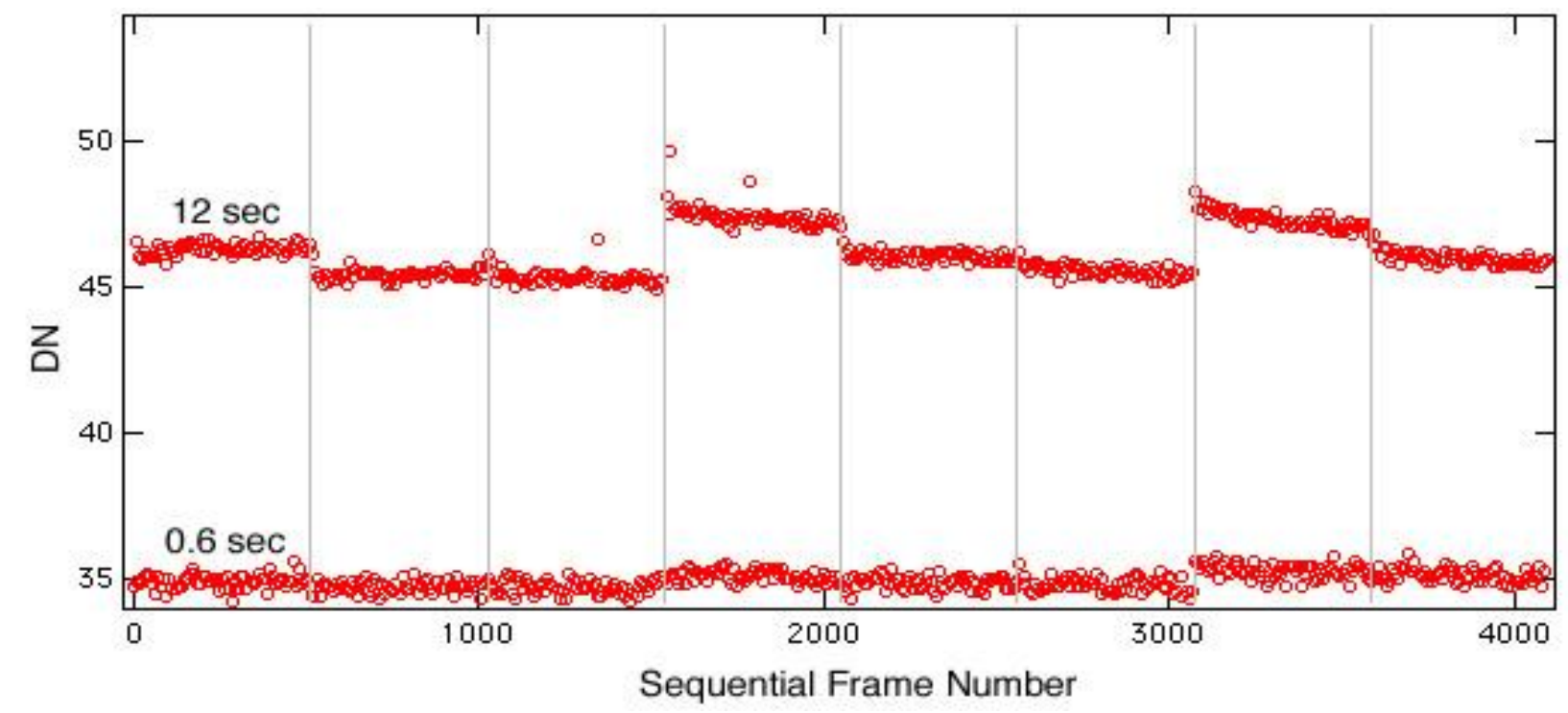

Figure 9. Median DN values of the frames. The horizontal axis is the frame number increasing sequentially in time. The light gray lines demarcate individual runs of the test sequence. 


\subsubsection{Photometric stability}

Another measurement made during the test was observations of the SWIR source in the center and four quadrants of channels 1 and 2. Approximately 25 images were taken in each filter at each array position. The flux was extracted using DAOPHOT in iraf, and an average value calculated for each of four sample times taken during the test. The results are shown in Figure 10. The measurements show the instrument response changing by up to 0.75 percent per day. This is probably an upper limit on the actual variations since we cannot separate out fluctuations in the SWIR lamp output. However, from this measured stability, it appears that our planned calibration star observation frequency of once per $12 \mathrm{hr}$ period will be sufficient to track the changes in the instrument responsivity, and meet the $2 \%$ relative photometric accuracy requirement.

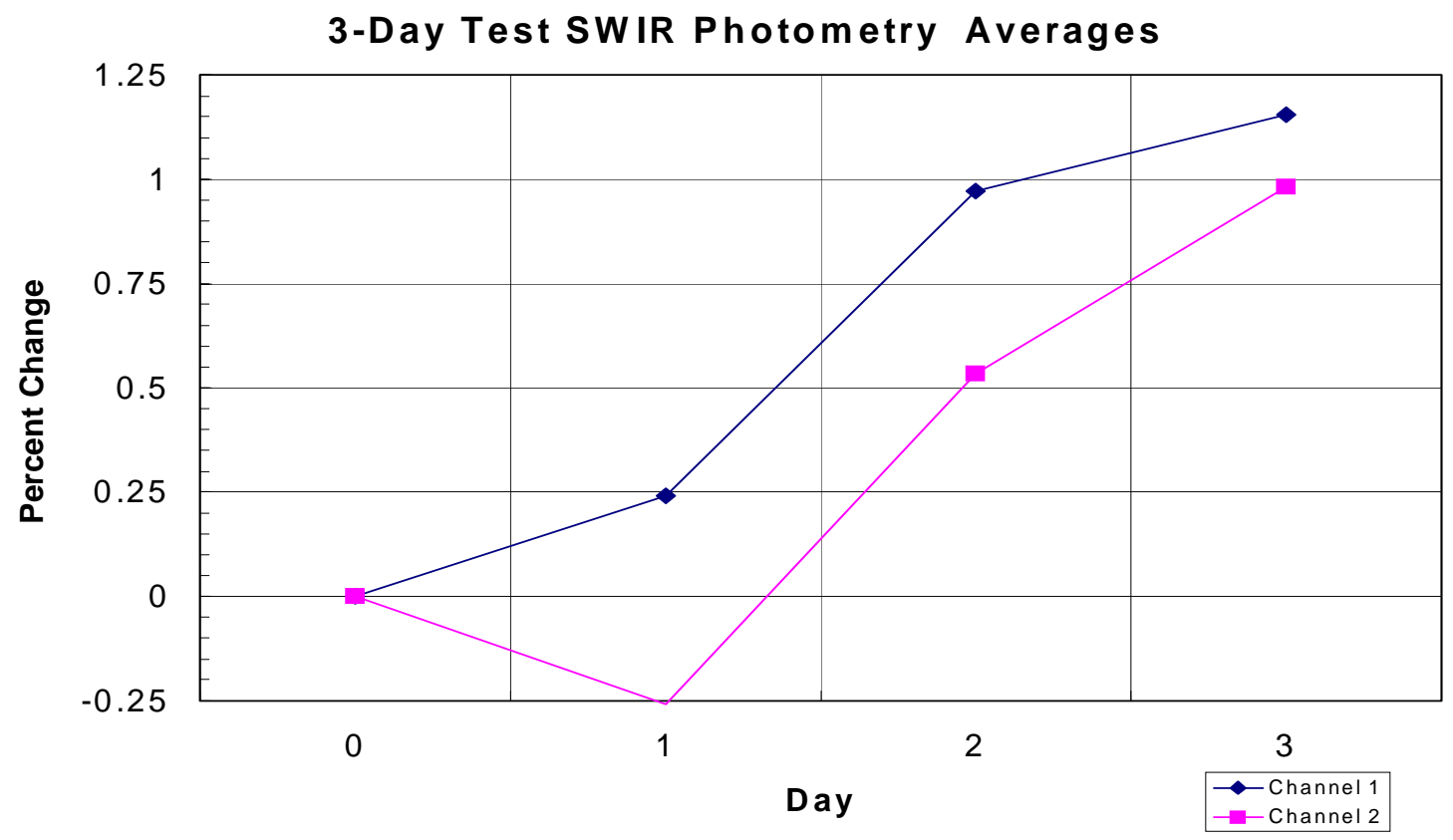

Figure 10. SWIR source photometry from 3-day stability test. The percent change relative to the initial sample are shown for channels 1 and 2 . Each point is the average photometry from all samples taken at that time.

\section{SUMMARY}

IRAC was successfully integrated into the MIC and the CTA at Ball Aerospace. A series of functional, verification, optical, and calibration tests were performed there. The tests showed that the instrument continues to function well and its projected sensitivity exceeds the project requirements. Many instrument behaviors were characterized and incorporated into the data reduction pipeline, which will improve the quality of the reduced data and allow a more effective use of IRAC during the SIRTF mission.

\section{ACKNOWLEDGEMENTS}

We acknowledge funding from NASA Office of Space Science for the work performed at the CfA and GSFC. We thank the Integration and Test group at GSFC for their excellent support of the IRAC testing program, including Ray Jungo, Ed Lander, Marty Brown, Ayman Mekhail, Maureen Armbruster, Peter Gorog, Jim MacLeod, and GSFC QA including Shirley Paul and Norm Lee. We also thank John Polizotti, Leslie Frazier, John Boczenowski, Warren Martell, and Paul Okun from SAO Central Engineering for system engineering and QA support. 


\section{REFERENCES}

1. G. G. Fazio, J. L. Hora, S. P. Willner, J. R. Stauffer, M. L. N. Ashby, Z. Wang, E. V. Tollestrup, J. Pipher, W. Forrest, C. McCreight, S. H. Moseley, W. F. Hoffmann, P. Eisenhardt, \& E. L. Wright, "The Infrared Array Camera (IRAC) for the Space Infrared Telescope Facility (SIRTF)", in Infrared Astronomical Instrumentation, ed. A. Fowler, Proc. SPIE 3354, pp. 1024-1031, 1998.

2. A. D. Estrada, G. Domingo, J. D. Garnett, A. W. Hoffman, N. A. Lum, P. J. Love, S. L. Solomon, J. E. Venzon, G. R. Chapman, K. D. Sparkman, C. McCreight, M. McKelvey, R. McMurray, J. Estrada, S. Zins, R. McHugh, \& R. Johnson, "Si:As IBC IR Focal Plane Arrays for Ground-based and Space-based Astronomy", in Infrared Astronomical Instrumentation, ed. A. M. Fowler, Proc. SPIE 3354, pp. 99-108, 1998.

3. J. Wu, W. J. Forrest, J. L. Pipher, N. Lum, \& A. Hoffman, "Development of infrared focal planes for space", Rev. Sci. Instrum., 68, pp. 3556-3578, 1997.

4. M. W. Werner, "SIRTF - An Overview", in Infrared Spaceborne Remote Sensing VIII, eds. M. Stojnik \& B. F. Andresen, Proc. SPIE, 4131, 1, 2000.

5. J. L. Hora, G. G. Fazio, S. P. Willner, M. L. N. Ashby, J.-S. Huang, S. T. Megeath, J. Stauffer, E.V. Tollestrup, Z. Wang, W. Glaccum, J. L. Pipher, W. J. Forrest, C. R. McCreight, M. McKelvey, W. F. Hoffmann, P. Eisenhardt, J. A. Surace, W. Reach, S. H. Moseley, R. Arendt, K. P. Stewart, F. D. Robinson, "Performance of the infrared array camera (IRAC) for SIRTF during instrument integration and test", in Infrared Spaceborne Remote Sensing VIII, eds. M. Stojnik \& B. F. Andresen, Proc. SPIE, 4131, 13, 2000.

6. J. L. Hora, G. G. Fazio, S. T. Megeath, S. P. Willner, M. L. N. Ashby, J.-S. Huang, Z. Wang, J. L. Pipher, W. J. Forrest, C. R. McCreight, M. McKelvey, R. McMurray, W. F. Hoffmann, P. Eisenhardt, J. R. Stauffer, W. Reach, J. A. Surace, W. Glaccum, S. H. Moseley, R. G. Arendt, K. P. Stewart, \& F. D. Robinson, "Calibration of the Infrared Array Camera (IRAC) for SIRTF", in The Calibration Legacy of the ISO Mission, VILSPA, Spain, February 2001, (ESA SP-481), in press, 2001.

7. F. L. Selden et al., "Implementation and testing of SIRTF's Infrared Array Camera (IRAC)", in Infrared Spaceborne Remote Sensing VIII, eds. M. Stojnik \& B. F. Andresen, Proc. SPIE, 4131, 50, 2000.

8. J. L. Fanson, G. G. Fazio, J. R. Houck, T. Kelly, G. H. Rieke, D. J. Tenerelli, M. Whitten, "Space Infrared Telescope Facility (SIRTF)", in Space Telescopes and Instruments V, eds. P. Y. Bely \& J. B. Breckinridge, Proc. SPIE, 3356, 478, 1998.

9. J. P. Schwenker, B. R. Brandl, W. F. Hoffmann, J. L. Hora, A. K. Mainzer, J. E. Mentzell, J. E. VanCleve, "SIRTFCTA Optical Performance Test Results", SPIE IR Space Telescopes and Instruments [4850-05] August 2002.

10. J. P. Schwenker, B. R. Brandl, W. L. Burmester, J. L. Hora, A. K. Mainzer, P. C. Quigley, J. E. VanCleve "SIRTFCTA Optical Performance Test", in IR Space Telescopes and Instruments, Proc. SPIE, [4850-48] August 2002.

11. W. F. Hoffmann, J. L. Hora, J. E. Mentzell, C. T. Marx, P. R. Eisenhardt, "Simfit and focus diversity: methods for determining the focus of a telescope without a focus slew", in IR Space Telescopes and Instruments, Proc. SPIE [4850-69], August 2002.

12. R. E. McMurray, Jr., R. R. Johnson, C. R. McCreight, M. E. McKelvey, J. D. Garnett, A. W. Hoffman, N. A. Lum, W. Y Lum, M. S. Smith, K. P. Sparkman, A. G. Toth, G. Domingo, D. J. Krebs, \& M. D. Jhabvala, "Si:As IBC array performance for SIRTF/IRAC", in Infrared Spaceborne Remote Sensing VIII, eds. M. Stojnik \& B. F. Andresen, Proc. SPIE, 4131, 62, 2000.

13. J. L. Pipher, W. J. Forrest, W. J. Glaccum, R. G. Benson, D. J. Krebs, M. D. Jhabvala, J.P. Rosbeck, N. A. Lum, W. Y. Lum, J. D. Garnett, A. W. Hoffman, \& G. Domingo, "InSb arrays for IRAC (InfraRed Array Camera) on SIRTF (Space Infrared Telescope Facility)", in Infrared Spaceborne Remote Sensing VIII, eds. M. Stojnik \& B. F. Andresen, Proc. SPIE, 4131, 7, 2000.

14. K. P. Stewart \& M. A. Quijada, "Cryo-transmittance and -reflectance of filters and beamsplitters for the SIRTF Infrared Array Camera", in Infrared Spaceborne Remote Sensing VIII, eds. M. Stojnik \& B. F. Andresen, Proc. SPIE, 4131, 218, 2000. 